\title{
Chromatic Roots of a Ring of Four Cliques
}

\author{
F.M. Dong* \\ Mathematics and Mathematics Education, National Institute of Education \\ Nanyang Technological University, Singapore \\ fengming.dong@nie.edu.sg \\ Gordon Royle \\ School of Mathematics \& Statistics \\ University of Western Australia, Australia \\ gordon@maths . uwa.edu. au \\ Dave Wagner \\ Department of Combinatorics and Optimization \\ University of Waterloo, Canada \\ dgwagner@math. uwaterloo.ca
}

Submitted: Mar 10, 2011; Accepted: Jul 8, 2011; Published: Jul 22, 2011

Mathematics Subject Classification: 05C15, 05C31,11C08, 65H04

\begin{abstract}
For any positive integers $a, b, c, d$, let $R_{a, b, c, d}$ be the graph obtained from the complete graphs $K_{a}, K_{b}, K_{c}$ and $K_{d}$ by adding edges joining every vertex in $K_{a}$ and $K_{c}$ to every vertex in $K_{b}$ and $K_{d}$. This paper shows that for arbitrary positive integers $a, b, c$ and $d$, every root of the chromatic polynomial of $R_{a, b, c, d}$ is either a real number or a non-real number with its real part equal to $(a+b+c+d-1) / 2$.
\end{abstract}

Keywords: graph, chromatic polynomial, chromatic root, ring of cliques

\section{Introduction}

A ring of cliques is a graph whose vertex set is the disjoint union of cliques, arranged in a cyclic order, such that the vertices of each clique are joined to all the vertices in the two neighbouring cliques. If the cliques have size $a_{1}, a_{2}, \ldots, a_{n}$ then we denote this graph by $R_{a_{1}, a_{2}, \ldots, a_{n}}$. Figure 1 shows the graph $R_{2,2,3,3}$.

\footnotetext{
${ }^{*}$ Corresponding author.
} 


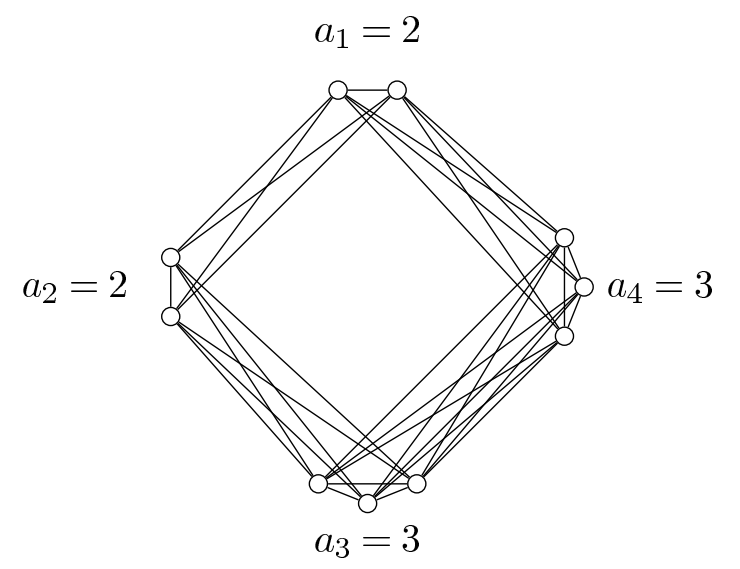

Figure 1: The graph $R_{2,2,3,3}$

Graphs with this structure have occurred several times previously in the study of chromatic polynomials and their roots. In particular, in proving that there are nonchordal graphs with integer chromatic roots, Read [6] considered the graphs in this family with $a_{1}=1$ (and he also used slightly different notation). Rings of cliques cropped up again recently in a preliminary investigation of the algebraic properties of chromatic roots (Cameron [1]) and in the course of this investigation, the chromatic roots of many of these graphs were computed. When the chromatic roots of the ring-of-clique graphs with exactly four cliques and a fixed number of vertices were plotted, an intriguing pattern was observed - all the non-real chromatic roots lie on a single vertical line. Figure 2 shows the union of the chromatic roots of the 12-vertex graphs of the form $R_{a, b, c, d}$.

Faced with such a striking empirically-observed pattern, we were led to explain it theoretically. This appears to require a surprisingly intricate argument, but eventually we obtain the following result:

Theorem 1 For arbitrary non-negative integers $a, b, c$ and $d$ the chromatic roots of $R_{a, b, c, d}$ are either real, or non-real with real part equal to $(a+b+c+d-1) / 2$.

The overall structure of the argument is as follows: $P\left(R_{a, b, c, d}, \lambda\right)$, the chromatic polynomial of $R_{a, b, c, d}$, is first expressed as the product of linear factors and a factor $Q_{a, b, c, d}(\lambda)$. It then suffices to show that the non-real roots of $Q_{a, b, c, d}(\lambda)$ all lie on the vertical line $\Re(\lambda)=(a+b+c+d-1) / 2$ in the complex $\lambda$-plane. Next the polynomial $F_{a, p, q, n}(z)$ is defined to be $Q_{a, b, c, d}(z+(a+b+c+d-1) / 2)$ thus translating the vertical line supposed to contain the roots to the imaginary axis and also reparameterizing the problem (in a somewhat counterintuitive way). Then $F_{a, p, q, n}$ is shown to be an even polynomial and we define a fourth polynomial $W_{a, p, q, n}$ by $W_{a, p, q, n}\left(z^{2}\right)=F_{a, p, q, n}(z)$. The proof is completed by demonstrating that $W_{a, p, q, n}$ is real-rooted using polynomial interleaving techniques, and therefore $F_{a, p, q, n}$ has only real or pure imaginary roots as required. 


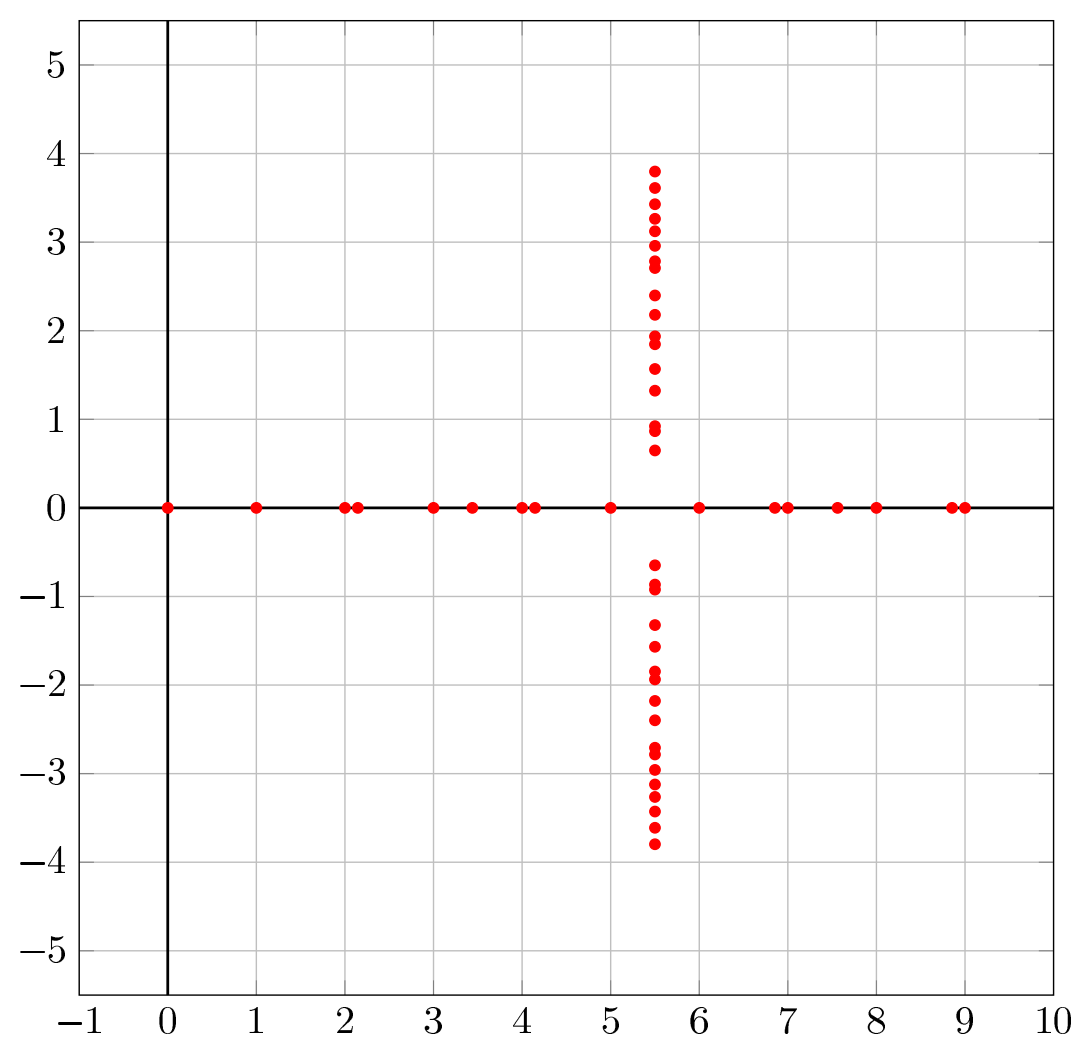

Figure 2: Chromatic roots of the graphs $R_{a, b, c, d}$ where $a+b+c+d=12$.

\section{Basics}

For any graph $G$ and any positive integer $\lambda$, let $P(G, \lambda)$ be the number of mappings $\phi$ from $V(G)$ to $\{1,2, \ldots, \lambda\}$ such that $\phi(u) \neq \phi(v)$ for every two adjacent vertices $u$ and $v$ in $G$. It is well-known that $P(G, \lambda)$ is a polynomial in $\lambda$, called the chromatic polynomial of $G$.

The chromatic polynomial of a graph $G$ has the following properties (see, for instance, $[3,5,7,9])$, which we will apply later.

Proposition 1 Let $G$ be a simple graph of order at least 2.

(i) If $u$ and $v$ are two non-adjacent vertices in $G$, then

$$
P(G, \lambda)=P(G+u v, \lambda)+P(G / u v, \lambda),
$$

where $G+u v$ is the graph obtained from $G$ by adding the edge joining $u$ and $v$, and $G /$ uv is the graph obtained from $G$ by identifying $u$ and $v$ and removing all parallel edges but one.

(ii) If $u$ is a vertex in $G$ which is adjacent to all other vertices in $G$, then

$$
P(G, \lambda)=\lambda P(G-u, \lambda-1),
$$


where $G-u$ is the graph obtained from $G$ by removing $u$.

If $a=0, R_{a, b, c, d}$ is a chordal graph and its chromatic polynomial is

$$
P\left(R_{0, b, c, d}, \lambda\right)=\frac{(\lambda)_{b+c}(\lambda)_{c+d}}{(\lambda)_{c}}
$$

and if $a \geq 1$ and $c \geq 1$, then applying Proposition 1 repeatedly yields that

$$
P\left(R_{a, b, c, d}, \lambda\right)=\lambda P\left(R_{a-1, b, c, d}, \lambda-1\right)+c \lambda P\left(R_{a-1, b, c-1, d}, \lambda-1\right) .
$$

For a non-negative integer $a$ and real numbers $b, c$ and $d$, define a polynomial $Q_{a, b, c, d}(z)$ in $z$ as follows: $Q_{0, b, c, d}(z)=1$ and for $a \geq 1$,

$$
Q_{a, b, c, d}(z)=(z-b-c)(z-c-d) Q_{a-1, b, c, d}(z-1)+c(z-a-c+1) Q_{a-1, b, c-1, d}(z-1) .
$$

It is clear that $Q_{a, b, c, d}(z)$ is a polynomial of order $2 a$ in $z$.

Proposition 2 Let $a, b, c$ and $d$ be any non-negative integers. Then

$$
P\left(R_{a, b, c, d}, \lambda\right)=\frac{(\lambda)_{b+c}(\lambda)_{c+d}}{(\lambda)_{a+c}} Q_{a, b, c, d}(\lambda)
$$

Proof. If $a=0$, then (6) follows from (3) and the definition of $Q_{a, b, c, d}(\lambda)$. Now assume that $a \geq 1$. By (4) and induction, we have

$$
\begin{aligned}
P\left(R_{a, b, c, d}, \lambda\right)= & \lambda P\left(R_{a-1, b, c, d}, \lambda-1\right)+c \lambda P\left(R_{a-1, b, c-1, d}, \lambda-1\right) \\
= & \lambda \frac{(\lambda-1)_{b+c}(\lambda-1)_{c+d}}{(\lambda-1)_{a+c-1}} Q_{a-1, b, c, d}(\lambda-1) \\
& +c \lambda \frac{(\lambda-1)_{b+c-1}(\lambda-1)_{c+d-1}}{(\lambda-1)_{a+c-2}} Q_{a-1, b, c-1, d}(\lambda-1) \\
= & \frac{(\lambda)_{b+c}(\lambda)_{c+d}}{(\lambda)_{a+c}}\left[(\lambda-b-c)(\lambda-c-d) Q_{a-1, b, c, d}(\lambda-1)\right. \\
& \left.+c(\lambda-a-c+1) Q_{a-1, b, c-1, d}(\lambda-1)\right] .
\end{aligned}
$$

The result then follows.

Define $\left(\begin{array}{l}x \\ 0\end{array}\right)=1$ and $\left(\begin{array}{l}x \\ r\end{array}\right)=x(x-1) \ldots(x-r+1) / r$ ! for any positive integer $r$ and any complex number $x$.

Proposition 3 For any non-negative integer $a$ and real numbers $b, c$ and $d$,

$$
Q_{a, b, c, d}(\lambda)=a ! \sum_{i=0}^{a} i !(a-i) !\left(\begin{array}{c}
c \\
i
\end{array}\right)\left(\begin{array}{c}
\lambda-b-c \\
a-i
\end{array}\right)\left(\begin{array}{c}
\lambda-c-d \\
a-i
\end{array}\right)\left(\begin{array}{c}
\lambda-a-c+i \\
i
\end{array}\right)
$$


Proof. It is trivial if $a=0$ as $Q_{0, b, c, d}(z)=1$. Now assume that $a \geq 1$. By (5) and induction,

$$
\begin{aligned}
& Q_{a, b, c, d}(\lambda) \\
& =(\lambda-b-c)(\lambda-c-d) Q_{a-1, b, c, d}(\lambda-1)+c(\lambda-a-c+1) Q_{a-1, b, c-1, d}(\lambda-1) \\
& =(\lambda-b-c)(\lambda-c-d)(a-1) ! \sum_{i=0}^{a-1}\left\{i !(a-i-1) !\left(\begin{array}{c}
c \\
i
\end{array}\right)\left(\begin{array}{c}
\lambda-b-c-1 \\
a-i-1
\end{array}\right)\right. \\
& \left.\left(\begin{array}{c}
\lambda-c-d-1 \\
a-i-1
\end{array}\right)\left(\begin{array}{c}
\lambda-a-c+i \\
i
\end{array}\right)\right\} \\
& +c(\lambda-a-c+1)(a-1) ! \sum_{i=0}^{a-1}\left\{i !(a-i-1) !\left(\begin{array}{c}
c-1 \\
i
\end{array}\right)\left(\begin{array}{c}
\lambda-b-c \\
a-i-1
\end{array}\right)\right. \\
& \left.\left(\begin{array}{c}
\lambda-c-d \\
a-i-1
\end{array}\right)\left(\begin{array}{c}
\lambda-a-c+i+1 \\
i
\end{array}\right)\right\} \\
& =(a-1) ! \sum_{i=0}^{a-1} i !(a-i) !(a-i)\left(\begin{array}{c}
c \\
i
\end{array}\right)\left(\begin{array}{c}
\lambda-b-c \\
a-i
\end{array}\right)\left(\begin{array}{c}
\lambda-c-d \\
a-i
\end{array}\right)\left(\begin{array}{c}
\lambda-a-c+i \\
i
\end{array}\right) \\
& +(a-1) ! \sum_{i=0}^{a-1}\left\{i !(a-i-1) !(i+1)^{2}\left(\begin{array}{c}
c \\
i+1
\end{array}\right)\left(\begin{array}{c}
\lambda-b-c \\
a-i-1
\end{array}\right)\right. \\
& \left.\left(\begin{array}{c}
\lambda-c-d \\
a-i-1
\end{array}\right)\left(\begin{array}{c}
\lambda-a-c+i+1 \\
i+1
\end{array}\right)\right\} \\
& =(a-1) ! \sum_{i=0}^{a-1} i !(a-i) !(a-i)\left(\begin{array}{c}
c \\
i
\end{array}\right)\left(\begin{array}{c}
\lambda-b-c \\
a-i
\end{array}\right)\left(\begin{array}{c}
\lambda-c-d \\
a-i
\end{array}\right)\left(\begin{array}{c}
\lambda-a-c+i \\
i
\end{array}\right) \\
& +(a-1) ! \sum_{i=1}^{a}(i-1) !(a-i) ! i^{2}\left(\begin{array}{c}
c \\
i
\end{array}\right)\left(\begin{array}{c}
\lambda-b-c \\
a-i
\end{array}\right)\left(\begin{array}{c}
\lambda-c-d \\
a-i
\end{array}\right)\left(\begin{array}{c}
\lambda-a-c+i \\
i
\end{array}\right) \\
& =a ! \sum_{i=0}^{a} i !(a-i) !\left(\begin{array}{c}
c \\
i
\end{array}\right)\left(\begin{array}{c}
\lambda-b-c \\
a-i
\end{array}\right)\left(\begin{array}{c}
\lambda-c-d \\
a-i
\end{array}\right)\left(\begin{array}{c}
\lambda-a-c+i \\
i
\end{array}\right) .
\end{aligned}
$$

The result then follows.

For any non-negative integer $a$ and real numbers $p, q, n$, define

$$
F_{a, p, q, n}(z)=a ! \sum_{i=0}^{a} i !(a-i) !\left(\begin{array}{c}
a+p+q-1 \\
i
\end{array}\right)\left(\begin{array}{c}
z+n+i-1 \\
i
\end{array}\right)\left(\begin{array}{c}
z-p \\
a-i
\end{array}\right)\left(\begin{array}{c}
z-q \\
a-i
\end{array}\right) \cdot(10
$$

Then (8) and (10) implies that $Q_{a, b, c, d}(z+(a+b+c+d-1) / 2)=F_{a, p, q, n}(z)$, where

$$
\left\{\begin{array}{l}
p=(b+c-a-d+1) / 2 \\
q=(c+d-a-b+1) / 2 \\
n=(b+d-a-c+1) / 2 .
\end{array}\right.
$$

In the next section, we shall show that $F_{a, p, q, n}(z)$ is an even polynomial in $z$, and the polynomial obtained from $F_{a, p, q, n}(z)$ by replacing $z^{2}$ by $z$ (i.e., $W_{a, p, q, n}(z)$ defined 
on Page 9) has only real roots for an arbitrary positive integer $a$ and arbitrary real numbers $p, q, n$ satisfying the condition that $p+q, p+n$ and $q+n$ are all non-negative (see Proposition 10). This result implies that every root of $F_{a, p, q, n}(z)$ is either a real number or a non-real number with its real part equal to 0 if $a$ is a positive integer and $p+q, p+n$ and $q+n$ are all non-negative real numbers. For arbitrary positive integers $a, b, c, d$, if $a \leq \min \{b, c, d\}$ and $p, q$ and $n$ are given in (11), then $p+q=c-a+1>0$, $p+n=b-a+1>0$ and $q+n=d-a+1>0$. Since $Q_{a, b, c, d}(z+(a+b+c+d-1) / 2)=$ $F_{a, p, q, n}(z)$, where $p, q$ and $n$ are given in (11), the following result is obtained.

Proposition 4 For arbitrary positive integers $a, b, c$ and $d$, if $a \leq \min \{b, c, d\}$, then every root of $Q_{a, b, c, d}(z)$ is either a real number or a non-real number with its real part equal to $(a+b+c+d-1) / 2$. Therefore, for arbitrary non-negative integers $a, b, c$ and $d$, every root of $P\left(R_{a, b, c, d}, \lambda\right)$ is either a real number or a non-real number with its real part equal to $(a+b+c+d-1) / 2$.

\section{The polynomial $F_{a, p, q, n}(z)$}

From the definition of $F_{a, p, q, n}(z)$, we have $F_{0, p, q, n}(z)=1$ and $F_{1, p, q, n}(z)=z^{2}+p q+p n+q n$. We shall show that $F_{a, p, q, n}(z)$ has a recursive expression in terms of $F_{a-1, p, q, n}(z)$ and $F_{a-2, p, q, n}(z)$. We first prove two properties of $F_{a, p, q, n}(z)$.

Proposition $5 \quad$ For any integer $a \geq 1$ and arbitrary real numbers $p, q, n$, if $p+q=0$, then

$$
F_{a, p, q, n}(z)=(z-p)(z-q) F_{a-1, p+1, q+1, n}(z)
$$

Proof. For $a \geq 1$,

$$
\begin{aligned}
F_{a, p, q, n}(z)= & a ! \sum_{i=0}^{a} i !(a-i) !\left(\begin{array}{c}
a-1 \\
i
\end{array}\right)\left(\begin{array}{c}
z+n+i-1 \\
i
\end{array}\right)\left(\begin{array}{c}
z-p \\
a-i
\end{array}\right)\left(\begin{array}{c}
z-q \\
a-i
\end{array}\right) \\
= & (z-p)(z-q)(a-1) ! \\
& \sum_{i=0}^{a-1} i !(a-1-i) !\left(\begin{array}{c}
a \\
i
\end{array}\right)\left(\begin{array}{c}
z+n+i-1 \\
i
\end{array}\right)\left(\begin{array}{c}
z-p-1 \\
a-1-i
\end{array}\right)\left(\begin{array}{c}
z-q-1 \\
a-1-i
\end{array}\right) \\
= & (z-p)(z-q) F_{a-1, p+1, q+1, n}(z) .
\end{aligned}
$$

Proposition 6 For any integer $a \geq 1$ and arbitrary real numbers $p, q, n$,

$$
F_{a, p+1, q, n}(z)-F_{a, p, q, n}(z)=a(a+n+q-1) F_{a-1, p+1, q, n}(z) .
$$


Proof. For $a \geq 1$,

$$
\begin{aligned}
& F_{a, p+1, q, n}(z)-F_{a, p, q, n}(z) \\
& =a ! \sum_{i=0}^{a}\left\{i !(a-i) !\left(\begin{array}{c}
z+n+i-1 \\
i
\end{array}\right)\left(\begin{array}{c}
z-q \\
a-i
\end{array}\right)\right. \\
& \left.\left(\left(\begin{array}{c}
a+p+q \\
i
\end{array}\right)\left(\begin{array}{c}
z-p-1 \\
a-i
\end{array}\right)-\left(\begin{array}{c}
a+p+q-1 \\
i
\end{array}\right)\left(\begin{array}{c}
z-p \\
a-i
\end{array}\right)\right)\right\} \\
& =a ! \sum_{i=0}^{a}\left\{i !(a-i) !\left(\begin{array}{c}
z+n+i-1 \\
i
\end{array}\right)\left(\begin{array}{c}
z-q \\
a-i
\end{array}\right)\right. \\
& \left.\left(\left(\begin{array}{c}
a+p+q-1 \\
i-1
\end{array}\right)\left(\begin{array}{c}
z-p-1 \\
a-i
\end{array}\right)-\left(\begin{array}{c}
a+p+q-1 \\
i
\end{array}\right)\left(\begin{array}{c}
z-p-1 \\
a-i-1
\end{array}\right)\right)\right\} \\
& =a ! \sum_{i=1}^{a} i !(a-i) !\left(\begin{array}{c}
z+n+i-1 \\
i
\end{array}\right)\left(\begin{array}{c}
z-q \\
a-i
\end{array}\right)\left(\begin{array}{c}
a+p+q-1 \\
i-1
\end{array}\right)\left(\begin{array}{c}
z-p-1 \\
a-i
\end{array}\right) \\
& -a ! \sum_{i=0}^{a-1} i !(a-i) !\left(\begin{array}{c}
z+n+i-1 \\
i
\end{array}\right)\left(\begin{array}{c}
z-q \\
a-i
\end{array}\right)\left(\begin{array}{c}
a+p+q-1 \\
i
\end{array}\right)\left(\begin{array}{c}
z-p-1 \\
a-i-1
\end{array}\right) \\
& =a ! \sum_{i=0}^{a-1}(i+1) !(a-i-1) !\left(\begin{array}{c}
z+n+i \\
i+1
\end{array}\right)\left(\begin{array}{c}
z-q \\
a-i-1
\end{array}\right)\left(\begin{array}{c}
a+p+q-1 \\
i
\end{array}\right)\left(\begin{array}{c}
z-p-1 \\
a-i-1
\end{array}\right) \\
& -a ! \sum_{i=0}^{a-1} i !(a-i) !\left(\begin{array}{c}
z+n+i-1 \\
i
\end{array}\right)\left(\begin{array}{c}
z-q \\
a-i
\end{array}\right)\left(\begin{array}{c}
a+p+q-1 \\
i
\end{array}\right)\left(\begin{array}{c}
z-p-1 \\
a-i-1
\end{array}\right) \\
& =(n+q+a-1) a ! \\
& \sum_{i=0}^{a-1} i !(a-1-i) !\left(\begin{array}{c}
z+n+i-1 \\
i
\end{array}\right)\left(\begin{array}{c}
z-q \\
a-1-i
\end{array}\right)\left(\begin{array}{c}
a+p+q-1 \\
i
\end{array}\right)\left(\begin{array}{c}
z-p-1 \\
a-i-1
\end{array}\right) \\
& =a(a+n+q-1) F_{a-1, p+1, q, n}(z) \text {. }
\end{aligned}
$$

Now we can prove that $F_{a, p, q, n}(z)$ can be expressed in terms of $F_{a-1, p, q, n}(z)$ and $F_{a-2, p, q, n}(z)$.

Proposition 7 Let $p, q, n$ be arbitrary real numbers. For any integer $a \geq 2$,

$$
\begin{aligned}
F_{a, p, q, n}(z)= & \left(z^{2}+(a-1)(2 p+2 q+2 n+2 a-3)+p q+p n+q n\right) F_{a-1, p, q, n}(z) \\
& -(a-1)(p+q+a-2)(q+n+a-2)(p+n+a-2) F_{a-2, p, q, n}(z) .(16
\end{aligned}
$$

Proof. $\quad$ By the definition of $F_{a, p, q, n}(z)$, we have $F_{0, p, q, n}(z)=1, F_{1, p, q, n}(z)=z^{2}+p q+p n+q n$ and

$$
\begin{aligned}
F_{2, p, q, n}(z)= & z^{4}+(2 q+2 p q+1+2 p n+2 p+2 q n+2 n) z^{2}+p q^{2}+p q \\
& +q n+q^{2} n+p^{2} q^{2}+p^{2} n^{2}+p^{2} q+4 p q n+p n^{2}+2 p^{2} q n
\end{aligned}
$$




$$
+p n+2 p q^{2} n+2 p q n^{2}+q n^{2}+q^{2} n^{2}+p^{2} n .
$$

Thus it can be verified that (16) holds when $a=2$.

Assume that (16) holds for every integer $2 \leq a<k$, where $k \geq 3$. Now consider the case that $a=k$.

By the definition of $F_{a, p, q, n}(z), F_{a, p, q, n}(z)$ is also a polynomial of order $a$ in $p$. Let $q, n, z$ be any fixed real numbers. If (16) holds for all numbers $p$ in the set $\{-q+r: r=$ $0,1,2, \ldots\}$, then the result is proven.

By assumption on $a,(16)$ holds for $F_{a-1,-q+1, q+1, n}(z)$ and thus

$$
\begin{aligned}
& F_{a-1,-q+1, q+1, n}(z) \\
= & \left(z^{2}-5 a+2 a n+2 a^{2}+3-2 n-q^{2}\right) F_{a-2,-q+1, q+1, n}(z) \\
& -(a-2)(a-1)(-q-2+n+a)(q-2+n+a) F_{a-3,-q+1, q+1, n}(z) .
\end{aligned}
$$

By Proposition 5, for any integer $m \geq 1$,

$$
F_{m,-q, q, n}(z)=\left(z^{2}-q^{2}\right) F_{m-1,-q+1, q+1, n}(z) .
$$

Hence

$$
\begin{aligned}
F_{a,-q, q, n}(z)= & \left(z^{2}-5 a+2 a n+2 a^{2}+3-2 n-q^{2}\right) F_{a-1,-q, q, n}(z) \\
& -(a-2)(a-1)(-q-2+n+a)(q-2+n+a) F_{a-2,-q, q, n}(z),
\end{aligned}
$$

implying that (16) holds for $F_{a,-q, q, n}(z)$.

In the remaining part of this proof, we shall show that if (16) holds for $F_{a, p, q, n}(z)$, then (16) holds for $F_{a, p+1, q, n}(z)$. Assume (16) holds for $F_{a, p, q, n}(z)$, and so

$$
\begin{aligned}
F_{a, p, q, n}(z)= & \left(z^{2}+(a-1)(2 p+2 q+2 n+2 a-3)+p q+p n+q n\right) F_{a-1, p, q, n}(z) \\
& -(a-1)(p+q+a-2)(q+n+a-2)(p+n+a-2) F_{a-2, p, q, n}(z) .(18)
\end{aligned}
$$

By assumption on $a$, (16) holds for $F_{a-1, p+1, q, n}(z)$ and so

$$
\begin{aligned}
& F_{a-1, p+1, q, n}(z) \\
= & \left(z^{2}+(a-2)(2 p+2 q+2 n+2 a-3)+(p+1)(n+q)+q n\right) F_{a-2, p+1, q, n}(z) \\
& -(a-2)(p+q+a-2)(q+n+a-3)(p+n+a-2) F_{a-3, p+1, q, n}(z) .
\end{aligned}
$$

By Proposition 6, (19) and (19), we have

$$
\begin{aligned}
& F_{a, p+1, q, n}(z) \\
= & F_{a, p, q, n}(z)+a(a+q+n-1) F_{a-1, p+1, q, n}(z) \\
= & \left(z^{2}+(a-1)(2 p+2 q+2 n+2 a-3)+p q+p n+q n\right) F_{a-1, p, q, n}(z) \\
& -(a-1)(p+q+a-2)(q+n+a-2)(p+n+a-2) F_{a-2, p, q, n}(z) \\
& +a(a+q+n-1) F_{a-1, p+1, q, n}(z) \\
= & \left(z^{2}+(a-1)(2 p+2 q+2 n+2 a-3)+p q+p n+q n\right)
\end{aligned}
$$




$$
\begin{aligned}
& \left(F_{a-1, p+1, q, n}(z)-(a-1)(a+q+n-2) F_{a-2, p+1, q, n}(z)\right) \\
& -(a-1)(p+q+a-2)(q+n+a-2)(p+n+a-2) \\
& \left(F_{a-2, p+1, q, n}(z)-(a-2)(a+q+n-3) F_{a-3, p+1, q, n}(z)\right) \\
& +a(a+q+n-1) F_{a-1, p+1, q, n}(z) \\
= & \left(z^{2}+(a-1)(2 p+2 q+2 n+2 a-3)+p q+p n+q n\right) \\
& \left(F_{a-1, p+1, q, n}(z)-(a-1)(a+q+n-2) F_{a-2, p+1, q, n}(z)\right) \\
& -(a-1)(p+q+a-2)(q+n+a-2)(p+n+a-2) F_{a-2, p+1, q, n}(z) \\
& +(a-1)(a+q+n-2)\left(-F_{a-1, p+1, q, n}(z)+\right. \\
& \left.\left(z^{2}+(a-2)(2 p+2 q+2 n+2 a-3)+(p+1)(n+q)+q n\right) F_{a-2, p+1, q, n}(z)\right) \\
& +a(a+q+n-1) F_{a-1, p+1, q, n}(z) \\
= & \left(z^{2}+(a-1)(2 p+2 q+2 n+2 a-1)+(p+1)(n+q)+q n\right) F_{a-1, p+1, q, n}(z) \\
& -(a-1)(p+q+a-1)(q+n+a-2)(p+n+a-1) F_{a-2, p+1, q, n}(z) .
\end{aligned}
$$

Thus (16) holds for $F_{a, p+1, q, n}(z)$. Hence (16) holds for $F_{a, p, q, n}(z)$ for all numbers $p$ in the set $\{q+r: r=0,1,2, \ldots\}$ and therefore the result is proved.

Since $F_{0, p, q, n}(z)=1$ and $F_{1, p, q, n}(z)=z^{2}+p q+p n+q n$, Proposition 7 implies that $F_{a, p, q, n}(z)$ is an even polynomial in $z$. For any non-negative integer $a$ and real numbers $p, q, n$, let $W_{a, p, q, n}(z)$ be the polynomial in $z$ defined as follows: $W_{0, p, q, n}(z)=1$, $W_{1, p, q, n}(z)=z+p q+p n+q n$ and for $a \geq 2$,

$$
\begin{aligned}
W_{a, p, q, n}(z)= & (z+(a-1)(2 p+2 q+2 n+2 a-3)+p q+p n+q n) W_{a-1, p, q, n}(z) \\
- & (a-1)(p+q+a-2)(q+n+a-2)(p+n+a-2) W_{a-2, p, q, n}(z) .
\end{aligned}
$$

Thus it is clear that $F_{a, p, q, n}(z)=W_{a, p, q, n}\left(z^{2}\right)$.

For two non-increasing sequences $\left(a_{1}, a_{2}, \ldots, a_{m}\right)$ and $\left(b_{1}, b_{2}, \ldots, b_{n}\right)$ of real numbers, we say the first interleaves the second if $m=n+1$ and $\left(a_{1}, b_{1}, a_{2}, b_{2}, \ldots, a_{n}, b_{n}, a_{n+1}\right)$ is an non-increasing sequence, or $m=n$ and $\left(a_{1}, b_{1}, a_{2}, b_{2}, \ldots, a_{n}, b_{n}\right)$ is an non-increasing sequence. If both polynomials $f(x)$ and $g(x)$ in $x$ with real coefficients have only real roots and the non-increasing sequence formed by all roots of $f(x)$ interleaves the nonincreasing sequence formed by all roots of $g(x)$, then we say $f(x)$ interleaves $g(x)$. We need to apply the following result (Proposition 8) given in Section 1.3 of [4]. Note that paper [8] has a result (Theorem 2.3 in that paper) stronger than Proposition 8. More details on polynomials with only real roots can be found in $[2,4,8]$.

Proposition 8 ([4]) Let $f(x)$ and $g(x)$ be polynomials with real coefficients and with positive leading coefficients and $u$ and $v$ be any real numbers. If $f(x)$ interleaves $g(x)$ and $v \leq 0$, then $(x-u) f(x)+v g(x)$ interleaves $f(x)$.

Applying Proposition 8 or Theorem 2.3 in [8], we can get the following result.

Proposition 9 Let a be any positive integer and $p, q, n$ be any real numbers.

(i) If $(p+q)(n+q)(n+p) \geq 0$, then $W_{2, p, q, n}(z)$ interleaves $W_{1, p, q, n}(z)$. 
(ii) If $a \geq 3,(p+q+a-2)(q+n+a-2)(p+n+a-2) \geq 0$ and $W_{a-1, p, q, n}(z)$ interleaves $W_{a-2, p, q, n}(z)$, then $W_{a, p, q, n}(z)$ interleaves $W_{a-1, p, q, n}(z)$.

Proof. By the definition of $W_{a, p, q, n}(z), W_{1, p, q, n}(z)=z+p q+p n+q n$ and

$$
\begin{aligned}
W_{2, p, q, n}(z)= & (z+2 p+2 q+2 n+1+p q+p n+q n)(z+p q+p n+q n) \\
& -(p+q)(q+n)(p+n) .
\end{aligned}
$$

As the only root of $W_{1, p, q, n}(z)$ is $-p q-p n-q n$ and $W_{2, p, q, n}(-p q-p n-q n)=-(p+$ $q)(n+q)(n+p) \leq 0, W_{2, p, q, n}(z)$ interleaves $W_{1, p, q, n}(z)$. So (i) holds.

By Proposition 7,

$$
\begin{aligned}
F_{a, p, q, n}(z)= & \left(z^{2}+(a-1)(2 p+2 q+2 n+2 a-3)+p q+p n+q n\right) F_{a-1, p, q, n}(z) \\
& -(a-1)(p+q+a-2)(q+n+a-2)(p+n+a-2) F_{a-2, p, q, n}(z) .
\end{aligned}
$$

Since $-(a-1)(p+q+a-2)(q+n+a-2)(p+n+a-2) \leq 0$ and $W_{a-1, p, q, n}(z)$ interleaves $W_{a-2, p, q, n}(z)$, Proposition 8 implies that $W_{a, p, q, n}(z)$ interleaves $W_{a-1, p, q, n}(z)$. Hence (ii) holds.

Notice that $W_{a, p, q, n}(z)=W_{a, q, p, n}(z)=W_{a, n, q, p}(z)$ holds for arbitrary real numbers $p, q, n$ and non-negative integer $a$, we assume that $p \leq q \leq n$ in the following.

Proposition 10 Let $p, q, n$ be arbitrary real numbers with $p \leq q \leq n$ and $p+q \geq 0$. Then, for every integer $a \geq 2, W_{a, p, q, n}(z)$ interleaves $W_{a-1, p, q, n}(z)$. Therefore, for every positive integer $a, W_{a, p, q, n}(z)$ has only real roots and every root of $F_{a, p, q, n}(z)$ is either a real number or a non-real number with its real part equal to 0.

Proof. Since $p+q \geq 0$ and $p \leq q \leq n$, we have $q+n \geq p+n \geq 0$ and so Proposition 9 (i) implies that $W_{2, p, q, n}(z)$ interleaves $W_{1, p, q, n}(z)$. Then, by Proposition 9 (ii), $W_{a, p, q, n}(z)$ interleaves $W_{a-1, p, q, n}(z)$ for every integer $a \geq 3$.

By the discussion immediately preceding Proposition 4, it follows that for all positive integers $a, b, c, d$ with $a \leq \min \{b, c, d\}$, the hypotheses of Proposition 10 are satisfied and hence we have proved Theorem 1.

Remark: There is another way to obtain the result of Proposition 10 by showing that all roots of $W_{a, p, q, n}(z)$ are actually the eigenvalues of a symmetric matrix with real entries only. Assume that $p, q, n$ are arbitrary real numbers with $p \leq q \leq n$ and $p+q \geq 0$. For any positive integer $a$, let $B_{a}=\left(b_{i, j}\right)$ be the $a \times a$ symmetric matrix whose non-zero entries are $b_{i, i}, b_{i, i-1}, b_{i-1, i}$ given below:

$$
b_{i, i}=-((i-1)(2 p+2 q+2 n+2 i-3)+p q+p n+q n)
$$

for all $i=1,2, \cdots, a$ and

$$
b_{i-1, i}=b_{i, i-1}=((i-1)(p+q+i-2)(q+n+i-2)(p+n+i-2))^{1 / 2}
$$

for all $i=2, \cdots, a$. It is not difficult to show that $\operatorname{det}\left(z I_{a}-B_{a}\right)=W_{a, p, q, n}(z)$ for all $a \geq 1$, where $I_{a}$ is the identity matrix of size $a$. Since $B_{a}$ is a symmetric matrix with real entries only, all roots of $\operatorname{det}\left(z I_{a}-B_{a}\right)$ are real and thus Proposition 10 follows. 


\section{Further properties of $F_{a, p, q, n}$ and $W_{a, p, q, n}$}

Even if $p+q<0$, there are still some situations in which $W_{a, p, q, n}(z)$ has only real roots. In this section we consider these, although they do not correspond to values of the parameters $a, p, q$ and $n$ that arise from rings of cliques. We need to apply the following result on the factorization of $F_{a, p, q, n}(z)$ when $a+p+n=1$ or $a+p+n=2$.

Proposition 11 Let $a$ be an integer with $a \geq 1$ and $p, q, n$ be arbitrary real numbers.

(i) If $a+p+n=1$, then

$$
F_{a, p, q, n}(z)=\prod_{j=0}^{a-1}\left(z^{2}-(n+j)^{2}\right)
$$

(ii) If $a+p+n=2$, then

$$
F_{a, p, q, n}(z)=\left(z^{2}+(p-1)(n-1)+a q\right) \prod_{j=0}^{a-2}\left(z^{2}-(n+j)^{2}\right) .
$$

Proof. (i) If $a+p+n=1$, then

$$
i !(a-i) !\left(\begin{array}{c}
z-p \\
a-i
\end{array}\right)\left(\begin{array}{c}
z+n+i-1 \\
i
\end{array}\right)=\prod_{j=0}^{a-1}(z+n+j) .
$$

Thus

$$
\begin{aligned}
F_{a, p, q, n}(z) & =a ! \sum_{i=0}^{a} i !(a-i) !\left(\begin{array}{c}
a+p+q-1 \\
i
\end{array}\right)\left(\begin{array}{c}
z-p \\
a-i
\end{array}\right)\left(\begin{array}{c}
z-q \\
a-i
\end{array}\right)\left(\begin{array}{c}
z+n+i-1 \\
i
\end{array}\right) \\
& =a ! \prod_{j=0}^{a-1}(z+n+j) \sum_{i=0}^{a}\left(\begin{array}{c}
a+p+q-1 \\
i
\end{array}\right)\left(\begin{array}{c}
z-q \\
a-i
\end{array}\right) \\
& =a ! \prod_{j=0}^{a-1}(z+n+j)\left(\begin{array}{c}
a+p+q-1+z-q \\
a
\end{array}\right) \\
& =a ! \prod_{j=0}^{a-1}(z+n+j)\left(\begin{array}{c}
z-n \\
a
\end{array}\right) \\
& =\prod_{j=0}^{a-1}\left(z^{2}-(n+j)^{2}\right) .
\end{aligned}
$$

Thus (i) holds.

(ii) Now let $a+p+n=2$. Since $F_{1, p, q, n}(z)=z^{2}+p q+p n+q n$, it is easy to verify that (ii) holds when $a=1$. Assume that (ii) holds for any integer $1 \leq a<k$, where $k \geq 2$. Now let $a=k$. 
Since $a+p+n=2$, by Proposition 7 ,

$$
F_{a, p, q, n}(z)=\left(z^{2}+(a-1)(2 p+2 q+2 n+2 a-3)+p q+p n+q n\right) F_{a-1, p, q, n}(z) .
$$

As $a-1+p+n=1$, by (i) of this result, we have

$$
F_{a-1, p, q, n}(z)=\prod_{j=0}^{a-2}\left(z^{2}-(n+j)^{2}\right) .
$$

Since $p+n+a=2$, it can be verified that

$$
(a-1)(2 p+2 q+2 n+2 a-3)+p q+p n+q n=(p-1)(n-1)+a q .
$$

Hence (ii) also holds.

Proposition 12 Let $p, q, n$ be arbitrary real numbers with $p \leq q \leq n$.

(i) If $p+q$ is a negative integer, then for every integer a with $a \geq 2-p-q, W_{a, p, q, n}(z)$ interleaves $W_{a-1, p, q, n}(z)$.

(ii) If $q+n$ is an integer, then for every integer a with $\max \{2,2-q-n\} \leq a \leq 2-p-n$, $W_{a, p, q, n}(z)$ interleaves $W_{a-1, p, q, n}(z)$.

Proof. (i) First consider the case that $a=2-p-q$. Since $p+q \leq-1$, we have $a \geq 3$. Proposition 11 implies that $W_{a, p, q, n}(z)$ interleaves $W_{a-1, p, q, n}(z)$.

Now assume that $a>2-p-q$ and $W_{a-1, p, q, n}(z)$ interleaves $W_{a-2, p, q, n}(z)$. Since $a>2-p-q$, we have $a+p+q-2 \geq 1$ and so $a+q+n-2 \geq a+p+n-2 \geq a+p+q-2 \geq 1$. Thus Proposition 9 (ii) implies that $W_{a, p, q, n}(z)$ interleaves $W_{a-1, p, q, n}(z)$. Therefore (i) holds.

(ii) The result is trivial if $\max \{2,2-q-n\}>2-p-n$. Now assume that $\max \{2,2-$ $q-n\} \leq 2-p-n$.

Let $a=\max \{2,2-q-n\}$. Then $a \geq 2-q-n$, implying that $a+q+n-2 \geq 0$. We also have $a \leq 2-p-n$, implying that $a+p+n-2 \leq 0$ and so $a+p+q-2 \leq 0$. If $a=\max \{2,2-q-n\}=2$, then Proposition 9 (i) implies that $W_{2, p, q, n}(z)$ interleaves $W_{1, p, q, n}(z)$, i.e., $W_{a, p, q, n}(z)$ interleaves $W_{a-1, p, q, n}(z)$. If $a=\max \{2,2-q-n\}=2-q-n$, then Proposition 11 implies that $W_{a, p, q, n}(z)$ interleaves $W_{a-1, p, q, n}(z)$.

Now assume that $\max \{2,2-q-n\}<a \leq 2-p-n$ and $W_{a-1, p, q, n}(z)$ interleaves $W_{a-2, p, q, n}(z)$. Note that $\max \{2,2-q-n\}<a \leq 2-p-n$ implies that $a+q+n-2>0$ and $a+p+q-2 \leq a+p+n-2 \leq 0$. Thus Proposition 9 (ii) implies that $W_{a, p, q, n}(z)$ interleaves $W_{a-1, p, q, n}(z)$. Therefore (ii) holds.

By Proposition 12, the following result is obtained.

Proposition 13 Let a be a positive integer and $p, q, n$ be arbitrary real numbers with $p \leq q \leq n$. If one of the following conditions holds, then $W_{a, p, q, n}(z)$ has only real roots and therefore every root of $F_{a, p, q, n}(z)$ is either a real number or a non-real number with its real part equal to 0 :

(i) $p+q$ is a negative integer and $a \geq 1-p-q$; and

(ii) $q+n$ is an integer and $\max \{1,1-q-n\} \leq a \leq 2-p-n$. 


\section{Acknowledgments}

We wish to thank the Isaac Newton Institute for Mathematical Sciences, University of Cambridge, for generous support during the programme on Combinatorics and Statistical Mechanics (January-June 2008), where this work was started and partially finished.

\section{References}

[1] Peter J. Cameron, Algebraic Properties of Chromatic Roots, Available online at www. maths. qmul.ac.uk/ pjc/csgnotes/alchrom1.pdf

[2] Maria Chudnovsky and Paul Seymour, The roots of the independence polynomial of a clawfree graph, J. Combin. Theory Ser. B 97 (2007), 350-357.

[3] F.M. Dong, K.M. Koh and K.L. Teo, Chromatic polynomials and chromaticity of graphs, World Scientific Publishing Co. Pte. Ltd., 2005.

[4] Steve Fisk, Polynomials, roots, and interlacing, arXiv:math/0612833v2.

[5] R.C. Read, An introduction to chromatic polynomials, J. Combin. Theory 4 (1968), $52-71$.

[6] R.C. Read, A large family of chromatic polynomials, in: Proceedings of the Third Caribbean Conference on Combinatorics and Computing (Bridgetown, 1981), (1981), 23-41.

[7] R.C. Read and W.T.Tutte, Chromatic polynomials, in: Selected Topics in Graph Theory III (eds. L.W.Beineke and R.J.Wilson), Academic Press, New York (1988), $15-42$.

[8] Lily L. Liu and Yi Wang, A unified approach to polynomial sequences with only real zeros, Advances in Applied Mathematics 38 (2007), 542-560.

[9] A.A. Zykov, On some properties of linear complexes, (Russian) Mat. Sbornik N.S. 24 (66) (1949), 163-188. 